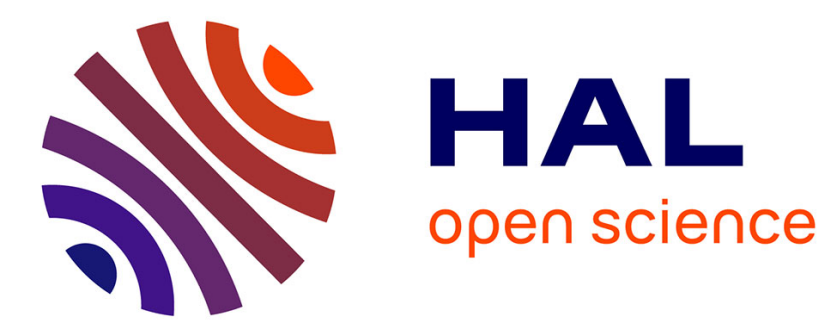

\title{
Gastric cancer in the elderly: An overview
}

\author{
W. Saif, N. Makrilia, A. Zalonis, M. Merikas, K. Syrigos
}

\section{To cite this version:}

W. Saif, N. Makrilia, A. Zalonis, M. Merikas, K. Syrigos. Gastric cancer in the elderly: An overview. EJSO - European Journal of Surgical Oncology, 2010, 36 (8), pp.709. 10.1016/j.ejso.2010.05.023 . hal-00608929

\section{HAL Id: hal-00608929 \\ https://hal.science/hal-00608929}

Submitted on 16 Jul 2011

HAL is a multi-disciplinary open access archive for the deposit and dissemination of scientific research documents, whether they are published or not. The documents may come from teaching and research institutions in France or abroad, or from public or private research centers.
L'archive ouverte pluridisciplinaire HAL, est destinée au dépôt et à la diffusion de documents scientifiques de niveau recherche, publiés ou non, émanant des établissements d'enseignement et de recherche français ou étrangers, des laboratoires publics ou privés. 


\section{Accepted Manuscript}

Title: Gastric cancer in the elderly: An overview

Authors: W. Saif, N. Makrilia, A. Zalonis, M. Merikas, K. Syrigos

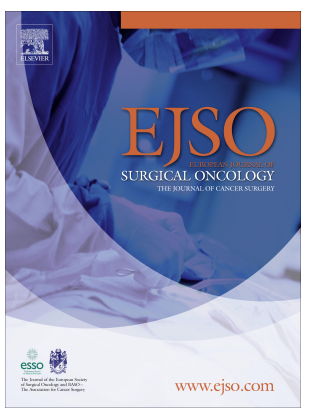

PII: S0748-7983(10)00131-9

DOI: $\quad$ 10.1016/j.ejso.2010.05.023

Reference: $\quad$ YEJSO 2985

To appear in: European Journal of Surgical Oncology

Received Date: 23 August 2009

Revised Date: 27 January 2010

Accepted Date: 17 May 2010

Please cite this article as: Saif W, Makrilia N, Zalonis A, Merikas M, Syrigos K. Gastric cancer in the elderly: An overview, European Journal of Surgical Oncology (2010), doi: 10.1016/j.ejso.2010.05.023

This is a PDF file of an unedited manuscript that has been accepted for publication. As a service to our customers we are providing this early version of the manuscript. The manuscript will undergo copyediting, typesetting, and review of the resulting proof before it is published in its final form. Please note that during the production process errors may be discovered which could affect the content, and all legal disclaimers that apply to the journal pertain. 
Review

Gastric cancer in the elderly: an overview

Running title: Gastric cancer in the elderly

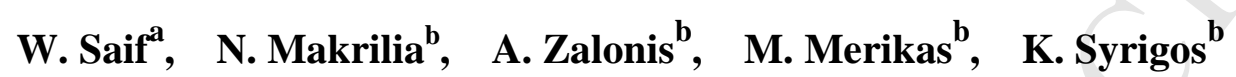

a. Department of Clinical Oncology, Yale University School of Medicine New Haven, CT.

b. Oncology Unit, $3^{\text {rd }}$ Department of Medicine, Sotiria General Hospital, Athens School of Medicine, Greece.

CORRESPONDENCE Address:

Konstantinos N. Syrigos, MD, PhD

Professor and Head, Oncology Unit

$3^{\text {rd }}$ Dept of Medicine, Athens University School of Medicine

Building Z, Sotiria General Hospital, Mesogion 152

11527 Athens, Greece

Tel: $\quad+302107475034$

Fax: + +30 2107781035

Email: knsyrigos@usa.net / ksyrigos@med.uoa.gr 


\section{Abstract}

Aims: Gastric cancer in the elderly represents a distinct entity with specific clinicopathological characteristics and the majority of affected patients belong to this age group. Subtotal or total gastrectomy with radical lymph node dissection, adjuvant chemo-radiotherapy or perioperative chemotherapy represent the only potentially curative treatment options and seem to be performed with acceptable morbidity and mortality rates in selected elderly patients. Published research is very limited due to the strict selection and under-representation of elderly patients in clinical trials. A review of current recommendations and practice was performed.

Methods: A comprehensive literature review was performed searching Medline for articles published since 1974, using "gastric cancer", "elderly" and "treatment" as key words.

Observations: The data suggest that elderly patients that fulfill the inclusion criteria of clinical trials experience the same advantages and toxicities from chemotherapy as younger patients. Fit elderly patients with operable gastric cancer should be canndidates for the standard surgical resection provided that pre-operative comorbidities are taken into account. Peri-operative chemotherapy or post-operative chemoradiotherapy should be added in case of locally advanced disease. Palliative systemic chemotherapy seems to prolong survival in recurrent and metastatic disease. Conclusions: Chronological age alone is not sufficient reason to withhold curative or palliative treatment from an elderly gastric cancer patient. Performance status does not suffice in order to estimate the general condition of elderly patients and cofactors regarding their functional, social and mental status have to be considered.

Word count (abstract): 234

Key words: Gastric cancer; Elderly; Treatment; Gastrectomy; Chemotherapy 


\section{Introduction}

Gastric cancer is a major health problem as it constitutes the second leading cause of cancer-related deaths. In Japan, Mexico and other Latin American countries, which are endemic areas, it is the most common gastrointestinal malignancy. It is considered a disease of the elderly, with its peak incidence occurring in the seventh decade of life. In the last decades, despite a plateau in the total number of patients, an increasing incidence of gastric cancer is being observed both in Japan and in USA, owing to the expanding life-span of the general population [1].

Many clinical oncologists are reluctant to have their elderly patients undergo the recommended treatment modalities, since they are at considerably higher risk of complications from surgery and/or chemo/radiotherapy. Furthermore, there is limited published data concerning gastric cancer treatment in this specific age group, due to the strict selection and under-representation of elderly patients in clinical trials. The scope of this review is to exhibit the distinguishing clinicopathological characteristics of gastric cancer in elderly patients and to clarify the treatment strategies by disease stage in this difficult to treat group of patients.

It is of importance to note that no standard definition of "elderly" exists, as different authors use the ages of $65,70,75$ and 80 years as thresholds. In this review, data were collected from studies published since 1974 referring to elderly patients, irrespective of age threshold. When the term "elderly" is used here-on in, we will be referring to patients over 75 years old, unless otherwise stated.

\section{A. Clinicopathological characteristics of gastric cancer in elderly population}

Gastric cancer of early and advanced stages in elderly patients exhibits a number of distinguishing characteristics. (Table 1)

\section{$\underline{\text { Gender }}$}

Data originating from both endemic and non-endemic areas show a male predominance in elderly gastric cancer patients but in young patients ( $<40$ years old), the gender ratio has constantly been reported to be approximately $1: 1$ [2] with a significant number of studies reporting a female predominance [2-3]. This sexual imbalance may reflect a more frequent and prolonged exposure of male elderly 
patients to environmental carcinogens. In addition, several investigators have reported a positive correlation between female gender and development of gastric cancer demonstrating that the presence of estrogen receptors is associated with poor survival in young ( $<40$ years old) patients $[4,5,6]$. The effect of sex hormones on gastric cancer development has been proposed as an explanation of the female predominance in that age group [7]. Several studies have even reported pregnancy as an adverse prognostic factor [8,9]. Recent changes in life style - raise of the percentage of active smokers among females - may also have contributed to the above gender ratio.

\section{Family history}

Only few studies have addressed the absence of positive family history in elderly gastric cancer patients [2] as compared to patients aged 45 years or less [3,10]. In Western countries, a hereditary type of diffuse, poorly-differentiated, infiltrative adenocarcinoma has been described in young patients. It is generated by a germline mutation of the CDH1 gene, which encodes the adhesion molecule E-cadherin, inherited by the autosomal dominant mode [11,12]. Still, most investigators believe that gastric cancer genetic predisposition observed in patients under the age of 40 involves multiple genes and environmental factors $[3,13]$.

\section{$\underline{\text { Tumor intragastic location }}$}

Regarding tumor intragastric location in elderly gastric cancer patients, the vast majority of studies have documented predominance of lower or distal third gastric location, independently of pathological stage [14-17]. The incidence of lower or distal third gastric cancer in the elderly varies between $42-63 \%$, which is significantly higher than that observed in younger patients (aged 65-74) $(31 \%-44 \%)$ [2,14,16-17]. In the latter, gastric cancer is more frequent in the middle and upper third of the stomach $[3,15]$.

\section{$\underline{\text { Macroscopic appearance }}$}

Macroscopic appearance of both early and advanced stage gastric cancer appears to be influenced by age. As defined by the Japanese classification of gastric carcinoma [18], the predominant type in early gastric cancer in elderly patients is the superficial depressed type IIc (accounting for approximately $46 \%$ of the cases), followed by the superficial elevated type IIa, and the polypoid type I (accounting cumulatively for 
$44 \%$ of cases) [14,17]. In younger patients (aged 65-74), the superficial depressed type is by far the most prevalent accounting for $90 \%$ of the cases [14,17]. In addition, increasing incidence of the superficial elevated type and decreasing incidence of the superficial depressed type has been observed with advancing age[14,17]. As far as advanced stage gastric carcinomas are concerned, according to Borrmann's classification [18], the most prevalent type in elderly patients, is the ulcerative type with border infiltration type III [14,15,17]. In younger patients (aged 65-74), the diffuse infiltration type IV accounts for more than $50 \%$ of the cases $[14,15,17]$. The former type of advanced stage gastric cancer constitutes a small but significant proportion of advanced cases among the elderly [17].

\section{Histological type}

Several studies have indicated that gastric cancer in elderly patients, irrespectively of tumor stage, is mainly well-differentiated [19-22], based on the criteria described by Ming [23] and Esaki [24]. Nevetheless, the pathological stage of the tumor had not been taken into consideration in these early reports [17]. Recent studies have revealed that the vast majority (up to $90 \%$ of cases) of early stage gastric cancers in the elderly $[14,17]$ are of the well differentiated type (mainly tubular and papillary adenocarcinomas), whereas poorly differentiated and signet ring cell carcinomas account for approximately only $10 \%$ of the cases [14,16-17]. In regard to advanced stage disease, it has been demonstrated that gastric cancer in the elderly exhibits more aggressive histological characteristics as compared to those observed in early stage disease [14,16-17]. The incidence of the predominantly well-differentiated carcinomas is almost equal to that of the predominantly poorly-differentiated $[14,17]$. However, the vast majority of the latter are found at the superficial site of welldifferentiated carcinomas[17]. Thus, gastric carcinomas in the elderly may principally develop as well-differentiated lesions which over time progress to poorly differentiated carcinomas, in contrast to those observed in younger age groups (aged 65-74), most of which emerge as poorly differentiated tumors from a very early phase[14,17].

\section{Synchronous carcinomas}

With regard to the incidence of multiple synchronous gastric carcinomas, several studies have demonstrated that they are more prevalent among elderly patients and 
that their incidence increases with advancing age [24-26]. In elderly patients, multiple gastric carcinomas account for almost 8\%-15\% of cases [24-26]. They are predominantly located in the lower third of the stomach, they are of elevated, welldifferentiated histological type and they present a tendency to collide and form single giant lesions [17,24-26]. Although their high prevalence in elderly patients has not fully been explained yet, a number of investigators have attributed it to the high incidence of intestinal type gastric cancer observed in that age group [24]. They suggested that intestinal type gastric cancer may be followed by multifocal carcinogenesis in stomachs with underlying atrophic gastritis.

\section{Patterns of metastases and recurrence}

Early studies had indicated that gastric cancer in elderly patients exhibits less metastasizing activity and that its pattern of metastases and recurrence is confined to the area around the primary focus: the upper abdomen including the liver [27]. Holmes and Hearne studied the relation between age and clinical stage of cancer progression in various types of cancer and reported no significant positive correlation between age and advanced clinical stage in gastric cancer [27]. However, these early reports did not take the histological type into consideration nor the pathological stage of gastric cancer. The glandular / well-differentiated / intestinal type is now considered the predominant histological type of gastric cancer in elderly patients $[14,17,28]$ and it generally tends to generate hematogenous metastases, predominantly to the liver via the portal vein [29-31]. Peritoneum invasion emerges infrequently [2930,32-33]. This distinct pattern of metastases and recurrence observed in the elderly may be related to the higher prevalence of blood vessels invasion observed in that age group [1,30]. Regarding the incidence of lymph node metastases, observations are controversial. Several reports have revealed no significant difference in the rate of lymph node involvement $[1,15,20,30]$, whereas few studies have demonstrated a trend toward higher incidence of lymph node metastases in the elderly, but not to a statistically significant extent [34]. In cases of early gastric cancer, numerous reports suggest a lower prevalence of lymph node metastases in the elderly as compared to younger patients (aged under 75) [14,16,35]. In addition, the examination of autopsy cases of fatal gastric cancer has confirmed the higher incidence of lymph node involvement in patients aged under 75 [28]. 


\section{B. Treatment modalities for gastric cancer in elderly patients}

In Japan and Europe, treatment guidelines for gastric cancer have been issued [36], and the standard therapeutic strategy by stage has been established. It is, however, important to develop treatment strategies for elderly gastric cancer patients taking multiple factors into account: distinguishing clinicopathological characteristics of gastric cancer, pre-treatment medical and nutritional status and early and long-term

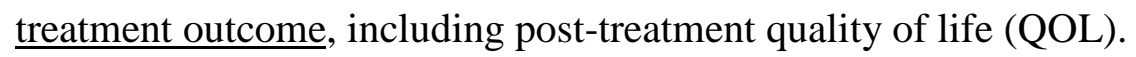

\section{Surgical treatment}

Surgical resection accompanied by dissection of a minimum of 14, and optimal 25 lymph nodes is the only modality that is potentially curative. It is recommended by the ESMO Guidelines Working Group for stages I-IV Mo [36]. However, many physicians are reluctant to have elderly patients undergo gastric surgery because of surgery risks, early and long term outcome, including postoperative quality of life, and life expectancy issues.

\section{i. Resection rate and curative resection rate}

\section{$\underline{\text { Early studies }}$}

Historically, early studies evaluating gastric cancer surgery in elderly patients reported extremely low curative resection rates [37-40]. Data from an English registry demonstrated that, during the 1957-1981 period, less than $20 \%$ of patients aged over 80 years received any form of surgical treatment, while the proportion of patients not receiving any treatment at all in that age group was as high as $87 \%$ [37]. The resection rate ranged from approximately $25 \%$ in patients aged $70-79$ years to almost $5 \%$ in patients over 80 , while the curative resection rates were $16 \%$ and $4 \%$, respectively [37]. Moreover, a Dutch population-based study conducted from 1982 to 1992 reported that the resection rate was $64 \%$ in patients under 59 years old and only $35 \%$ in those aged over 80 years [38]. In the late 1970s, two Italian hospital series reported almost $50 \%$ resectability in patients over 80 years old [39-40].

\section{$\underline{\text { Recent changes in resection rates }}$}


Since then, the widespread availability of fiberoptic endoscopy, which leads to earlier diagnosis of gastric cancer [41], combined with improved surgical and anesthetic techniques, have resulted in a gradual but significant increase of the resection and curative resection rates in the elderly. A French population-based series reported an increase in resectability from 31\% in 1976 - 1978 to $53 \%$ in 1991 - 1993 in elder patients [41], while data from a Japanese surgical department showed a rise of the resection rate from $56 \%$ in 1965 - 1970 to $83 \%$ in 1986 - 1990 in the same age group [30]. The Dutch D1 D2 Trial evaluated D1 and D2 lymph node dissection after curative resection in patients aged under 85 years and observed no significant differences in resection rates between five age groups [42], while a Japanese single hospital series, conducted from 1971 to 1990, reported a 89\% resection rate and a curative resection rate as high as $76 \%$ in octogenarians patients [43]. Japanese and Far East studies report resection rates ranging from $72 \%$ [44] to $88 \%$ [45] and curative resection rates ranging from 52\% [46] to 77\% [44] in patients over 80 years old. More specifically, in the Kitamura et al study, resection rates were similar in all three age groups studied: $>80$ years old, 60-79 years old and $40-59$ years old (88\% vs $94 \%$ vs 98\%, respectively) [45]. In the Hanazaki et al trial, the curative resectability rate was statistically different between octagenerians and patients younger than 60 years old (52\% vs $75 \%$, respectively) [46]. European reports exhibit resection rates between $56.6 \%[47]$ to $93 \%$ [48] and curative resection rates ranging from $70 \%$ [49] to $91 \%$ [47]. In the Saiki et al trial, $75 \%$ of patients over 70 years old underwent subtotal gastrectomy and $25 \%$ of them had total gastrectomy. There was no statistically significant difference from patients younger than 70 [49].

\section{ii. Preoperative concomitant disorders}

Several studies have demonstrated that surgical risk, evaluated preoperatively with the American Society of Anesthesiologists (ASA) score, is significantly higher in elderly gastric cancer patients, mainly because of the higher rate of concomitant diseases [4748]. Katai et al [43] observed that the 90-day postoperative mortality rate was higher in octogenarian patients with pre-existing co-morbidities, especially in the subgroups of elderly patients undergoing total gastrectomy and D2 lymphadenectony. However, two contemporary European studies demonstrated that, even though the preoperative ASA risk was significantly higher in elderly gastric cancer patients, it was not correlated to postoperative morbidity and mortality nor to curative resection rates [47- 
48]. In the Coniglio et al trial, 35\%, 14\%. $26 \%$ and $25 \%$ of octogenarians had stage I, II, II and IV gastric cancer, respectively. The resection rate was 57\% whereas the curative resection rate was $83 \%$. When examining the TNM variables, the curative resection rates were $100 \%, 100 \%, 93 \%$ and $33 \%$ for T1, T2, T3 and T4 cancers, respectively. For N0, N1, N2 and N3 cancers, the curative resection rate was 100\%, $89 \%, 86 \%$ and 50\%, respectively [47]. In the Orsenigo et al study, with respect to tumor stage, $45 \%$ of elderly patients had stage III-IV gastric cancer and there was no statistically significant difference between the two age groups (over and under 75 years old). $55 \%$ of elderly patients underwent resection with D2 or more lymph node dissection and the curative resection rate was $93 \%$ [48].

\section{iii. Postoperative morbidity and mortality}

During the past decades, advances in surgical and anesthetic techniques coupled with improved perioperative intensive care have resulted in a significantly decline of the postoperative morbidity and mortality rate in elderly gastric cancer patients. Many published trials present similar rates of postoperative morbidity and mortality between octogenarians and younger than 80 gastric cancer patients.

\section{$\underline{\text { Japanese and Far East studies }}$}

Two Japanese studies have reported 18\% [44] and 32\% [43] rates of postoperative complications in curative and non-curative resection in octogenarians gastric cancer patients. The operative mortality rates in these studies were similar. Katai et al reported similar death rates during operation in octogenarians and younger than 80 years old patients without pre - existing morbidity, even though overall operative mortality in the elder age group was slightly higher (3\% vs 1\%, respectively) [43]. Kubota et al observed a higher, but statistically not significant, postoperative complications rate in octogenarian patients with curatively resected cancer and a postoperative mortality rate similar to that of patients aged 40-79 [50]. Kunisaki et al noted higher incidence of postoperative morbidity in the elderly among all registered patients-curatively and palliative resected [51]. Even though the incidence of postoperative morbidity was higher in the elderly among all patients who had undergone palliative resection, no statistically significant difference in the incidence of postoperative morbidity was reported between the two age cohorts among patients who had undergone curative resection [51]. With regard to postoperative mortality, no 
significant difference was observed between elderly and middle-aged patients, after palliative or curative resection [51]. In elderly patients, especially in those with palliative resection, the prevalence of postoperative morbidity is correlated to the incidence of preoperative concomitant disorders [51]. The same observation was made by Hora et al, who evaluated treatment strategies for gastric cancer in the elderly according to the number of abnormal parameters on preoperative examination [52]. They reported an increase in the incidence of postoperative complications according to the number of preoperative abnormal parameters. Overall postoperative morbidity rate was $37 \%$ in the elder vs $11 \%$ in younger patients. Overall hospital mortality rate in the elder patients was also significantly higher than in the younger ones (29\% vs $11 \%$, respectively), while the difference between the two age groups disappeared among patients with abnormalities in four or fewer items [52]. On the contrary, a Chinese study reported significantly higher postoperative morbidity and mortality rates in the elderly [29]. In the stepwise logistic regression analysis, age over 74 years was an independent risk factor affecting mortality [29].

\section{European and American studies}

An Italian study reported a $29 \%$ rate of postoperative morbidity after gastric cancer resection in patients aged over 75 years [48]. The postoperative morbidity in that age group was similar to that observed in younger patients, whether related to surgical complications or to medical pathologies [48]. The rate of postoperative mortality was $3 \%$ in both age groups. TNM stage and the number of positive lymph nodes were found to be independent risk factors for postoperative mortality [48]. Cuniglio et al reported similar rates of postoperative morbidity and mortality after curative gastric cancer resection in octogenarians as compared to patients aged less than 80, respectively [47]. Finally, a USA study by Saidi et al demonstrated identical overall postoperative morbidity rate after curative resection in cancer patients aged over 70 years as well as in patients younger than 70, 33\% in both age groups [49]. Postoperative mortality rate was slightly higher in the elderly ( $8 \%$ vs $4 \%$, respectively), but the difference was not statistically significant [49].

After taking the aforementioned studies into account, it seems that postoperative complication rates are higher in the elderly and depend greatly on the number and severity of pre-existing concomitant disorders. Morbidity after palliative surgery 
seems be higher in the elderly but in case of curative resection, this rate seems to drop to the levels observed in patients under 75 . Nevertheless, most studies agree that postoperative mortality rates are similar in these two age groups. These data need to be confirmed in large clinical trials that will study the association of these rates with preexisting comorbidities and tumor location more thoroughly.

\section{iv. Surgical procedures: Type of gastric surgery (subtotal versus total gastrectomy), dissection of adjacent organs (pancreatosplenectomy) and extent of lymphadenectomy}

\section{Type of gastric resection}

The dominant trend among surgeons is to perform subtotal gastrectomy in elderly patients $[43,46,48]$, since total gastrectomy in this age group had been associated with higher rates of postoperative morbidity and mortality as compared to subtotal gastrectomy [29,43,53]. The Dutch D1 D2 study univariate analysis determined that patients over the age of 65 submitted to total gastrectomy had a relative risk of 2.15 for hospital death and 3.25 for surgical complications, as compared to those who had undergone partial gastrectomy. In multivariate analysis, the risk disappeared since the type of resection was strongly related to the level of node dissection [53]. Katai et al demonstrated that total gastrectomy in octogenarian patients was associated with higher operative and 90-day mortality, especially in those with preoperative comorbidity [43].

\section{Dissection of adjacent organs}

Two Far East studies have demonstrated that splenectomy and combined resections of adjacent organs are associated with higher morbidity and mortality rates in elderly patients and are less frequently performed in this age group [29]. On the contrary, Kubota et al [50] reported that octagenarian patients can tolerate aggressive surgical procedures such as combined dissection of adjacent organs with the surgical results being comparable to those observed in younger patients (ages 40-79).

\section{Extent of lymphadenectomy}

With regard to the extent of lymph node resection, the vast majority of studies reported that extended - D2 or greater - lymphadenectomy is significantly less 
frequently performed in patients over the age of 80 , with the reported percentages ranging from $33 \%$ to $81 \%$ [49], whereas super - extended, D3 or greater, lymphadenectomy is not performed in this age group [47]. Egushi et al [54] reported that extended lymphadenectomy in elderly patients did not positively influence the 5year survival, while it resulted in higher mortality (10\% vs 1\%) and morbidity rate (57\% vs $27 \%$ ) as compared to limited lymphadenectomy. On the contrary, Kubota et al [50] demonstrated that standard D2 lymphadenectomy can be safely employed in octogenarian gastric cancer patients resulting in better loco-regional control of the disease without negatively affecting postoperative morbidity and mortality [50].

\section{v. Survival}

Overall mortality from gastric cancer has declined over the last few decades. Contemporary data from studies published during the last 15 years have revealed that in Japan and Far East countries, which are endemic areas of gastric cancer, the overall 5 -year survival rate after curative resection in octogenarian patients varies between $44 \%$ [43] to $65 \%$ [46], while disease-specific 5-year survival rates vary from 53\% [50] to $62.5 \%$ [43]. In palliative resection, no differences in overall and disease-specific survival have been observed between age groups 65-74 and >74 [29]. Western studies have reported comparable results. Tumor stage, lymph node metastasis and depth of invasion of primary tumor have been shown to be independent prognostic factor negatively affecting survival [50]. Thus, standard surgical treatment seems to be feasible even in elderly gastric cancer patients with pre - existing morbidity, offering possible long-term survival, whereas in elderly patients with poor medical status less aggressive surgical procedures should be applied.

\section{Multimodality treatment for locally advanced gastric cancer in elderly patients}

R0 resection represents the only treatment modality offering possible long-term survival. Nevertheless, a significant percentage will develop local-regional and/or distant recurrence [50]. Adjuvant chemoradiotherapy and perioperative chemotherapy are recommended by the ESMO Guidelines Working Group in an attempt to improve survival after curative resection in high-risk gastric cancer patients [36]. 
European and American trials

The Gastric Surgical Adjuvant Trial compared surgery plus postoperative chemoradiotherapy to surgery alone for curatively resected adenocarcinoma of the stomach or gastroesophageal junction [55]. Patients receiving post-operative chemotherapy with fluorouracil plus leucovorin combined with radiotherapy exhibited significantly better median overall survival and longer relapse-free survival. Age as a potential covariate yielded no significant differences to the effects of treatment. MacDonald et al [55] concluded that adjuvant chemoradiotherapy should be offered to all high risk gastric cancer patients and this treatment approach is considered to be standard therapy in the U.S. of America [36]. It should be pointed out though, that in this trial, $54 \%$ and $36 \%$ of patients had undergone a D0 and D1 dissection, respectively, whereas only $10 \%$ had undergone an extensive D2 dissection. This should be taken into account when comparing the above conclusions to those of other studies, since the standard surgical procedure in Japan is a D2 dissection with resection of all perigastric lymph nodes and some celiac, splenic or splenic-hilar, hepatic-artery and cardial lymph nodes, depending on the exact tumor location.

In most of the UK and other parts of Europe, perioperative chemotherapy has been adopted as standard of care [36]. The MAGIC trial randomly assigned 503 patients to receive three cycles of pre- and postoperative epirubicin, cisplatin and continuous I.V. infusion of 5-fluorouracil or surgery alone [56]. $20 \%$ of patients at the perioperative chemotherapy arm were over 70 years old. Improved overall survival and progression-free survival were demonstrated [56].

\section{$\underline{\text { Japanese trials }}$}

An important randomized multicenter phase III Japanese trial studying the benefits of adjuvant chemotherapy is the Adjuvant Chemotherapy Trial of S-1 for Gastric Cancer (ACTS-GC) study. This large trial randomly assigned 1059 patients with stage II and III gastric cancer to undergo gastrectomy with extended D2 lumph-node dissection followed by S-1 adjuvant chemotherapy for 1 year or to undergo surgery alone. In the first interim analysis, there was a statistically significant difference in overall and relapse-free survival in favor of the group receiving adjuvant chemotherapy and the trial was discontinued. Analysis of follow-up data showed that the 3-year overall survival was $80 \%$ vs $70 \%$ in the S-1 and surgery-only groups, respectively. Following these results published in 2007, adjuvant S-1 after curative surgery has become a 
standard treatment in Japan. Results concerning elderly patients were not statistically significant [57]. Various randomized trials are underway and their results are anticipated with great interest. The Stomach Cancer Adjuvant Multi-Institutional Group (SAMIT) trial is a currently active phase III trial that has enrolled 1480 patients with T3-T4 N0-2 P0 M0 gastric cancer. These patients have undergone macroscopically curative gastrectomy with D2 or comparable lymph-node resection. The study is planning to examine the survival benefit of sequential use of paclitaxel followed by oral fluoropyrimidines in comparison with fluoropyrimidines alone. Patients have been randomized with a two-by-two factorial design in 4 groups, receiving UFT alone (control), S1 alone, sequential paclitaxel-UFT and sequential paclitaxel-S1, respectively. This study expects that adding paclitaxel to a fluoropyrimidine may reduce peritoneal recurrence. The results of the SAMIT trial are expected to be disclosed in 2010 [58].

The impact of use of any scheme of multimodality treatment in the elderly can not be clearly evaluated since trials conducted so far have not reached any definite conclusions regarding this age group. There is need for more phase III clinical trials that are based on elderly patients. Currently, adjuvant chemotherapy alone is usually not recommended in Europe, since it offers small survival benefit with considerable toxicity [36]. Therefore, there seem to be two viable options for fit elderly patients with gastric cancer that is operable or that has been treated by surgical resection: preand postoperative chemotherapy or postoperative chemoradiotherapy, respectively.

\section{Treatment modalities for recurrent or metastatic gastric cancer in elderly patients.}

Palliative systemic chemotherapy offers survival advantages and better quality of life than best supportive care alone and therefore, represents the recommended treatment modality [36]. In elderly patients there is uncertainty regarding the extent of systemic palliative chemotherapy that should be offered.

\section{European trials}

The first study concerning efficacy and tolerability of palliative chemotherapy that included elderly patients with advanced oesophagogastric cancer was conducted between 1992 and 2001 [59]. 1080 eligible patients (24\% of participants were over 70 
years old) were assigned to receive either a platinum-containing regimen - ECF [epirubicin and cisplatin plus protracted venous infused 5-fluorouracil (PVI 5-FU)], or MCF [mitomycin C, cisplatin and PVI 5-FU], or PVI 5-FU alone or in combination with mitomycin $\mathrm{C}$ or FAMXT [methotrexate and 5-FU followed by doxorubicin]. In both age cohorts, platinum-containing regimens (ECF, MCF) achieved superior FFS (6.9 months in patients $<70$ years vs 7.2 months in those aged $>70$ years) and OS (8.8 months vs 7.9 months, respectively) as compared to PVI 5-FU +/- MMC (FFS:3.0 months vs 4.4 months, respectively, and OS: 5.2 months vs 6.6 months, respectively), and FAMXT (FFS:3.5 months vs 2.8 months, respectively, and OS: 6.1 months vs 5.0 months, respectively). Patients $\geq 70$ and $<70$ years of age had similar benefits from palliative chemotherapy without increased toxicities [59].

The Belgian phase III V325 trial compared docetaxel and cisplatin plus 5-fluorouracil (DCF) with ciplatin and 5-fluorouracil (CF) as first-line therapy for advanced gastric cancer [60]. DCF resulted in prolonged median time-to-progression (5.6 months vs 3.7 months. Interestingly, despite the higher incidence of toxicity observed with DCF, global health status (QOL evaluation) and Karnofsky performance status (clinical benefit evaluation) were preserved for a longer time. DCF should be reserved only for fit elderly patients [60].

The REAL-2 (Randomised ECF for Advanced Esophagogasric Cancer 2) trial evaluated capecitabine and oxaliplatin as alternatives to infused fluorouracil and cisplatin, respectively, of the ECF triplet regimen for untreated advanced oesophagogastric cancer [61]. This trial did not provide data solely from elderly patients as it included patients aged 22-83 years old. The trial tested for non-inferiority between ECF [epirubicin, cisplatin and PVI 5-FU], ECX [epirubicin, cisplatin and capecitabine], EOF [ epirubicin, oxaliplatin and PVI 5-FU] and EOX [ epirubicin, oxaliplatin and capecitabine]. Progression-free survival and response rates did not differ significantly among the regimens, while overall survival was longer with EOX than with ECF (11.2 months vs 9.9 months, respectively, $p=0.02)[61]$.

The Arbeitsgemeinschaft Internistiche Onkologie study evaluated fluorouracil and leucovorin plus either oxaliplatin (FLO) or cisplatin (FLP) for metastatic gastroesophageal carcinoma [62]. In patients aged over 65 FLO resulted in significantly superior response rates $(41 \%$ vs $17 \%$, respectively; $\mathrm{p}=0.012)$, progression-free survival (6.0 months vs 3.1 months, respectively; $\mathrm{p}=0.029$ ) and in an improved overall survival (OS) (13.9 months vs 7.2 months, respectively) as 
compared to FLP [62]. To our knowledge, the latter figure represents the highest median overall survival ever observed in that age group of patients in a phase III trial.

\section{$\underline{\text { Japanese and Far East trials }}$}

The first study of palliative chemotherapy specifically designed for patients over the age of 65 with advanced gastric cancer was performed in Korea and the results were presented at the ASCO 2006 meeting [63]. Chemotherapy consisted of oxaliplatin, folinic acid and 5-FU and a total of 24 patients were enrolled. Overall response rate was $50 \%$. Median progression-free and median overall survival were 5.4 months (95\% CI: 5.1 - 5.8 months) and 7.4 months (95\% CI: 4.4 - 10.4 months), respectively. The oxaliplatin/5-FU/FA regimen demonstrated satisfactory efficacy with an acceptable toxicity profile in patients with advanced or recurrent/metastatic gastric cancer in this age group [63].

A recent Chinese study evaluated the modified FOLFOX-4 regimen in a phase II/III trial as first-line chemotherapy for elderly patients with advanced gastric cancer [64]. A total of 44 patients aged 65 years or older were administered oxaliplatin, leucovorin and 5-fluorouracil on a 2-week cycle. Overall response rate was 53\%. The reported median time-to-progression and median overall survival rates were 6.5 months and 10.0 months, respectively. The modified FOLFOX-4 regimen proved to be active as well as well-tolerated in these patients [64].

In Japan, S-1, an oral anticancer drug that combines tegafur (a prodrug of fluorouracil) with 5-chloro-2,4-dihydropyrimidine (an antagonist of the rate-limiting enzyme for the degradation of fluorouracil ) and potassium oxonate (which blocks the phosphorylation of fluorouracil in the gastrointestinal tract decreasing gastrointestinal toxic effects) is considered the standard of care for advanced or recurrent/metastatic gastric cancer either alone or in combination with cisplatin [65]. Recently, a singlecenter Korean study evaluated the efficacy and safety of oral fluoropyrimidines plus cisplatin as 1st line treatment for patients aged over 70 with advanced gastric cancer [66]. Results concerning response rate, overall survival and toxicity were similar to those reported from previous studies regarding S-1 and cisplatin therapy in patients of all ages with metastatic gastric cancer [65] and superior to those reported in abstractform on capecitabine vs S-1 1st line monotherapy in patients aged over 65 with recurrent or metastatic gastric cancer [67]. 
To date, there is no single global standard regimen for the treatment of advanced gastric cancer [36]. Further ongoing studies, such as the international First-Line Advanced Gastric Cancer Study [68] are anticipated in order to establish the role of oral fluoropyrimidines plus cisplatin combination in the treatment of advanced gastric cancer.

\section{Conclusion}

Data in literature regarding elderly patients with gastric cancer seem to be limited and sometimes conflicting. Most studies though seem to agree that fit elderly patients with operable gastric cancer should be candidates for the recommended standard extensive surgical resection accompanied by resection of at least 14 lymph nodes, provided that pre-existing comorbidities and tumor location are considered. Patients with operable locally advanced disease should also be submitted to perioperative chemotherapy or postoperative chemoradiotherapy. In case of recurrent and metastatic disease, palliative systemic chemotherapy should be considered since it offers prolonged survival and preserves quality of life. In conclusion, data clearly show that age alone does not suffice in order to estimate the general performance status of an elderly patient and its eligibility for curative or palliative treatment. In the future, large phase III clinical trials studying treatment in elderly patients should be conducted, taking their various physiological profiles into account.

Conflict of interest statement: None

There were no study sponsors.

Ethics (Helsinki declaration): Conformed

Word count: 5204 
Table 1: Distinguishing clinicopathological characteristics of gastric cancer in elderly patients

1. Gender ratio

Male predominance (male to female ratio: $1.6: 1$ - 2.45:1)

2. Family history

Absence of positive family history

3. Tumor intragastric location

Predominantly lower or distal third location

4. Macroscopic appearance (varies according to disease stage)

-Early stage disease: superficial depressed (IIc) the predominant type

-Advanced stage disease: ulcerative with border infiltration (III) the predominant type

5. Histological type (varies according to disease stage)

-Early stage disease: well-differentiated

-Advanced stage disease: poorly-differentiated with well-

differentiated components at the superficial site

6. Synchronous carcinomas

Increased incidence of multiple synchronous carcinomas

7. Pattern of tumor growth, metastases, and recurrence

Liver the dominant metastatic and recurrence site; peritoneum involvement and lymph nodes metastases less frequently observed 


\section{REFERENCES}

1. Saito H, Osaki T, Murakami D, et al. Effect of age on prognosis in patients with gastric cancer. ANZ J Surg 2006;76:458-61.

2. Medina-Franco H, Heslin MJ, Cortes-Gonzalez R. Clinicopathological characteristics of gastric carcinoma in young and elderly patients: A comparative study. Ann Surg Oncol 2000;7:515-19.

3. Kunisaki C, Akiyama H, Nomura M, et al. Clinicopatological features of gastric carcinoma in younger and middle-aged patients: A comparative study. J Gastrointest Surg 2006;10:1023-32.

4. Tokunaga A, Nishi K, Matsukura N, et al. Estrogen and progesterone receptors in gastric cancer. Cancer 1986;57:1376-79.

5. Harrison JD, Jones Ja, Ellis IO, et al. Oestrogen receptor D5 antibody is an independent negative prognostic factor in gastric cancer. Br J Surg 1991; 78:334-36.

6. Kurita H. Clinico-epidemiological study of stomach cancer considering sex and age differences. Jpn J Cancer Clin 1974;20:580-93.

7. Furukawa $\mathrm{H}$, Iwanaga $\mathrm{T}$, Koyama $\mathrm{H}$, et al. Effects of sex hormones on carcinogenesis in the stomach of rats. Cancer Res 1982; 42:5181-82.

8. Furukawa H, Iwanaga T, Hiratsuka M, et al. Gastric cancer in young adults: Growth accelerating effect of pregnancy and delivery. J Surg Oncol 1994; 55:3-6.

9. Maeta M, Yamashiro H, Oka A, et al. Gastric cancer in the young with special reference to 14 pregnancy-associated cases: analysis based on 2,325 consecutive cases of gastric cancer. J Surg Oncol 1995; 58:191-95.

10. Santoro R, Carboni F, Lepiane P, et al. Clinicopathological features and prognosis of gastric cancer in young European adults. Br J Surg 2007;94:737-42.

11. Huntsman DG, Carreiro F, Lewis Fr, et al. Early gastric cancer in young, asymptomatic carriers of germ-line E-cadherin mutations. N Engl J Med 2001; 344:1904-09.

12. Caldas C, Carneiro F, Lynch HT, et al. Familial gastric cancer: overview and guidelines for management. J Med Genet 1999;36:873-80.

13.Tahara E. Molecular biology of gastric cancer. World J Surg 1995; 19: 484-90. 
14. Arai T, Esaki Y, Inoshita N, et al. Pathologic characteristics of gastric cancer in the elderly: a retrospective study of 994 surgical patients. Gastric Cancer 2004;7:1549.

15. Kim DY, Joo JK, Ryu SY, et al. Clinicopathologic characteristics of gastric carcinoma in elderly patients: A comparison with young patients. World $\mathrm{J}$ Gastroenterol 2005;11(1):22-26.

16. Ishigami S, Natsugoe S, Saihara T, et al. Clinical and pathologic features of early gastric cancer in elderly patients. Hepatogastroenterology 1997;44(16):1164-8.

17. Inoshita N, Yanagisawa A, Arai T, et al. Pathological characteristics of gastric carcinomas in the very old. Jpn J Cancer Res 1998;89:1087-92.

18. Japanese Gastric Cancer Association. Japanese classification of gastric carcinoma. $2^{\text {nd }}$ English Ed. Gastric Cancer 1998;1:10-24.

19. Fujiya T, Endoh K, Mikuni J, et al. Clinicopathological characteristics and surgical outcome of gastric carcinoma with regard to aging. Jpn J Gastroenterol Surg 1997;30:1699-05.

20. Kitamura K, Yamaguchi T, Taniguchi H, et al.:Clinicopathological characteristics of gastric cancer in the elderly. Br J Cancer 1996;73(6):798-802.

21. Kunisaki C, Kobayashi S, Kido Y, et al. Clinicopathological evaluation of senile patients with gastric cancer. Jpn J Clin Surg 1996; 57:1831-37.

22. Sho M, Imagawa A, Hosoi T, et al. Clinical study of surgical cases of gastric cancer in patients over 80 years old. Jpn J Soc Clin Surg 1996;57:1838-43.

23. Ming S. Gastric carcinoma. A pathological classification. Cancer 1977;39:247585.

24. Esaki Y, Hirokawa K, Yamashiro M. Multiple gastric cancers in the aged with special reference to intramucosal cancers. Cancer 1987;59:560-5.

25. Kitamura K, Yamaguchi T, Okamoto K, et al. Clinicopathologic features of synchronous multifocal early gastric cancers. Anticancer Res 1997;17(1B): 643-46.

26. Mitsudomi T, Watanabe A, Matsusaka T, et al. A clinicopathological study of synchronous multiple gastric cancer. Br J Cancer 1989; 76(3): 237-40.

27. Holmes FF, Hearne E III. Cancer Stage-to-stage relationship: Implications for cancer screening in the elderly. J Am Geriatr Soc 1981;29: 55-57.

28. Esaki Y, Hirayama R, Hirokawa K. A comparison of patterns of metastasis in gastric cancer by histologic type and age. Cancer 1990;65:2086-90. 
29. Wu CW, Lo SS, Shen KH, Hsieh MC, Lui WY, P'eng FK. Surgical mortality, survival and quality of life after resection for gastric cancer in the elderly. World $\mathbf{J}$ Surg 2000;24:465-72.

30. Maehara Y, Emi Y, Tomisaki S, et al. Age-related characteristics of gastric carcinoma in young and elderly patients. Cancer 1996;77(9):1774-80.

31. Nielsen J, Aagaard J, Toftgaard C. Gastric cancer with special reference to prognostic factors: A review of 779 cases. Acta Chir Scand 1985;151:49-55.

32. Goseki N. [The relationship between the histological type of gastric carcinoma and the mode of spreading.] Stomach Intest 1983;18:643-49.

33. Okuno K, Shigeoka H, Tanaka A, et al. Clinicopathological evaluation of T2gastric cancer among age groups. Hepatogastroenterology 2000;47:1180-82.

34. Wang JY, Hsieh JS, Huang CJ, et al. Clinicopathologic study of advanced gastric cancer without serosal invasion in young and old patients. J Surg Oncol 1996; 63(1):36-40.

35. Fujimoto S, Takahashi M, Ohkubo H, et al. Comparative clinicopathologic features of early gastric cancer in young and older patients. Surgery 1994;115(4):51620.

36. Cunningham D, Oliveira J; ESMO Guidelines Working Group. Gastric cancer: ESMO clinical recommendations for diagnosis, treatment and follow-up. Ann Oncol 2008;19(Suppl 2):ii23-ii24.

37. Winslet MC, Mohsen YMA, Powell J. The influence of age on the surgical management of carcinoma of the stomach. Eur J Surg Oncol 1996;22:220-224.

38. Damhuis RA, Tilanus HW. The influence of age on resection rates and postoperative mortality in 2773 patients with gastric cancer. Eur J Cancer 1995;31A: 928-31.

39. Coluccia C, Ricci EB, Martzola GG, et al. Gastric cancer in the elderly: results of surgical treatment. Int Surg 1987;72: 4-10.

40. Roviello F, Marrelli D, De Stefano A, et al. Complications after surgery for gastric cancer in patients aged 80 years and over. Jpn J Clin Oncol 1998;28:116-22.

41. Benhamiche AM, Faivre J, Tazi AM, et al. Time trends in diagnostic strategy, treatment, and prognosis of gastric cancer in the elderly: a population based study. Eur J Cancer Prev 1997;6:71-77. 
42. Bonenkamp JJ, Songun I, Hermans J, et al. Randomised comparison of morbidity after D1 and D2 dissection for gastric cancer in 996 Dutch patients. Lancet 1995;345:745-48.

43. Katai H, Sasako M, Sano M, Maruyama K. The outcome of surgical treatment for gastric carcinoma in the elderly. Jpn J Clin Oncol 1998;28:112-5.

44. Eguchi T, Fujii M, Takayama T. Mortality for gastric cancer in elderly patients. J Surg Oncol 2003;84:132-6.

45. Kitamura K, Sugimachi K, Saku M. Evaluation of surgical treatment for patients with gastric cancer who are over 80 years of age. Hepatogastroenterology 1999;46: 2074-80.

46. Hanazaki K, Wakabayashi M, Sodeyama H, et al. Surgery for gastric cancer in patients older than 80 years of age. Hepatogastroenterology 1998;45:268-75.

47. Coniglio A, Tiberio GA, Busti M, et al. Surgical treatment for gastric carcinoma in the elderly. J Surg Oncol 2004;88:201-5.

48. Orsenigo E, Tomajer V, Palo SD, et al. Impact of age on postoperative outcomes in 1118 gastric cancer patients undergoing surgical treatment. Gastric Cancer 2007;10:39-44.

49. Saidi RF, Bell JL, Dudrick PS. Surgical resection for gastric cancer in elderly patients: is there a difference in outcome? J Surg Res 2004;118:15-20.

50. Kubota H, Kotoh T, Dhar DK, et al. Gastric resection in the aged ( $>$ or $=80$ years) with gastric carcinoma: a multivariate analysis of prognostic factors. Aust N Z J Surg 2000;70:254-7.

51. Kunisaki C, Akiyama H, Nomura M, et al. Comparison of surgical outcomes of gastric cancer in elderly and middle-aged patients. Am J Surg 2006;191(2): 216-24.

52. Hara H, Isozaki H, Nomura E, et al. Evaluation of treatment strategies for gastric cancer in the elderly according to the number of abnormal parameters on preoperative examination. Jpn J Surg 1999; 29: 837-41.

53. Sasako M. Risk factors for surgical treatment in the Dutch. Gastric Cancer Trial. Br J Surg 1997;84:1567-71.

54. Eguchi T, Takahashi Y, Ikarashi M, Kasahara M, Fujii M. Is extended lymph node dissection necessary for gastric cancer in elderly patients? Eur J Surg 2000;166:94953. 
55. MacDonald JS, Smalley SR, Benedetti J, et al. Chemoradiotherapy after surgery compared with surgery alone for adenocarcinoma of the stomach or gastroesophageal junction. N Engl J Med 2001;345:725-30.

56. Cunningham D, Allum WH, Stenning SP, et al. Perioperative chemotherapy versus surgery alone for respectable gastroesophageal cancer. New Engl J Med 2006;355:11-20.

57. Sakuramoto S, Sasako M, Yamaguchi T, et al. Adjuvant chemotherapy for gastric cancer with S-1, an oral fluoropyrimidine. N Engl J Med 2007;357(18):1810-20.

58. Tsuburaya A, Sakamoto J, Morita S, et al. A randomized phase III trial of postoperative adjuvant oral fluoropyrimidine versus sequential paclitaxel/oral fluoropyrimidine; and UFT versus S1 for T3/T4 gastric carcinoma: the Stomach Cancer Adjuvant Multi-institutional Trial Group (Samit) Trial. Jpn J Clin Oncol 2005;35(11)672-5.

59. Trumper M, Ross PJ, Cunningham D, et al. Efficacy and tolerability of chemotherapy in elderly patients with advanced oesophago-gastric cancer: A pooled analysis of three clinical trials. Eur J Cancer 2006;42:827-34.

60. Van Cutsem E, Moiseyenko VM, Tjulandin S, et al. Phase III study of docetaxel and cisplatin plus fluorouracil compared with cisplatin and fluorouracil as first-line therapy for advanced gastric cancer: A report of the V325 study group. J Clin Oncol 2006;24:4991-97.

61. Cunningham D, Starling N, Rao S, et al. Capecitabine and oxaliplatin for advanced esophagogastric cancer. New Engl J Med 2008;358:36-46.

62. Al-Batran SE, Hartmann JT, Probst S, et al. Phase III trial in metastatic gastroesophageal adenocarcinoma with fluorouracil, leucovorin plus either oxaliplatin or cisplatin: a study of the Arbeitsgemeinschaft Internistische Onkologie. J Clin Oncol 2008;26: 1435-42.

63. Choi I, Lee K, Oh D, et al. Oxaliplatin, 5-fluorouracil, and folic acid as first-line chemotherapy for elderly patients with advanced gastric cancer. J Clin Oncol 2006;24(18S):14155.

64. Liu ZF, Guo QS, Zhang XQ, et al. Biweekly oxaliplatin in combination with continuous infusional 5-fluorouracil and leucovorin (modified FOLFOX-4 regimen) as first-line chemotherapy for elderly patients with advanced gastric cancer. Am J Clin Oncol 2008;31:259-63. 
65. Koizumi W, Nahara H, Hara T, et al. S-1 plus cisplatin versus S-1 alone for firstline treatment of advanced gastric cancer (SPIRITS trial): A phase III trial. Lancet Oncol 2008;9:215-21.

66. Seol YM, Song MK, Choi YJ, et al. Oral fluoropyrimidines (Capecitabine or S-1) and cisplatin as first line treatment in elderly patients with advanced gastric cancer: a retrospective study. Jpn J Clin Oncol 2009;39:43-8.

67. Kang Y, Lee J, Min Y. A randomized multi-center phase II trial of capecitabine (X) versus S-1 (S) as first-line treatment in elderly patients with metastatic or recurrent unresectable gastric cancer. J Clin Oncol 2007;25:18S (Abstract 4546)

68. Ilson DH. Docetaxel, cisplatin, and fluorouracil in gastric cancer: does the punishment fit the crime? J Clin Oncol 2007;25:3188-90. 
Table 1: Distinguishing clinicopathological characteristics of gastric cancer in elderly patients

1. Gender ratio

Male predominance (male to female ratio: $1.6: 1$ - 2.45:1)

2. Family history

Absence of positive family history

3. Tumor intragastric location

Predominantly lower or distal third location

4. Macroscopic appearance (varies according to disease stage)

-Early stage disease: superficial depressed (IIc) the predominant type

-Advanced stage disease: ulcerative with border infiltration (III) the predominant type

5. Histological type (varies according to disease stage)

-Early stage disease: well-differentiated

-Advanced stage disease: poorly-differentiated with well-

differentiated components at the superficial site

6. Synchronous carcinomas

Increased incidence of multiple synchronous carcinomas

7. Pattern of tumor growth, metastases, and recurrence

Liver the dominant metastatic and recurrence site; peritoneum involvement and lymph nodes metastases less frequently observed 